\title{
Organic matter depositional microenvironment in deltaic channel deposits of Mahanadi river, Andhra Pradesh
}

\author{
Anjum Farooqui", T. Karuna Karudu ${ }^{1}$, D. Rajasekhara $\operatorname{Reddy}^{1}$ and Ravi Mishra ${ }^{2}$ \\ Birbal Sahni Institute of Palaeobotany, 53, University Road, Lucknow, INDIA \\ ${ }^{1}$ Delta Studies Institute, Andhra University, Sivajipalem, Visakhapatnam-17, INDIA \\ ${ }^{2}$ ONGC, 9, Kaulagarh Road, Dehra dun, INDIA \\ *Corresponding author. E-mail: afarooqui_2000@yahoo.com
}

\begin{abstract}
Quantitative and qualitative variations in microscopic plant organic matter assemblages and its preservation state in deltaic channel deposits of Mahanadi River was correlated with the depositional environment in the ecosystem in order to prepare a modern analogue for use in palaeoenvironment studies. For this, palynological and palynofacies study was carried out in 57 surface sediment samples from Birupa river System, Kathjodi-Debi River system and Kuakhai River System constituting Upper, Middle and Lower Deltaic part of Mahanadi river. The apex of the delta shows dominance of Spirogyra algae indicating high nutrient, low energy shallow ecosystem during most of the year and recharged only during monsoons. The depositional environment is anoxic to dysoxic in the central and south-eastern part of the Middle Deltaic Plain (MDP) and Lower Deltaic Plain (LDP) indicated by high percentage of nearby palynomorphs, Particulate Organic Matter (POM) and algal or fungal spores. The northern part of the delta show high POM preservation only in the estuarine area in LDP but high Amorphous Organic Matter (MOA) in MDP. The sediment here is deposited under dysoxic to oxic fluvial conditions. Thus, the monsoon intensity, direction of fluvial discharge, and the landward extent of sea water incursion through river mouths inducing bottom water salinity play an important role in defining the magnitude of POM and its preservation in the shallow Mahanadi deltaic ecosystem.
\end{abstract}

Keywords: Palynology, Palynofacies, Mahanadi delta, Surface sediment

\section{INTRODUCTION}

Degradation, preservation and compositional variation of sedimentary organic matter are controlled by sedimentary environments and sea-level position. River deltas are formed due to dynamic interaction of fluvial and marine agencies in the land- ocean interaction zone. Delta building varies depending upon geologic, tectonic, geographic, climatic and environmental settings. Palynofacies analysis involves the study of organic matter sedimentology whereby, total particulate organic matter (POM) such as fragments of wood, cuticle and palynomorphs such as pollen, spores and dinoflagellate cysts helps to understand the deltaic depositional microenvironment useful in palaeoclimatic and palaeoecological interpretations. Various factors play a role in the preservation of organic matter during sedimentation and burial, for example, anoxia, sediment size, primary productivity, organic source material, and contemporaneous diagenesis in the water column (Demaison and Moore, 1980; Emerson, et al., 1985; and Meyers, 1997). The key control in organic matter preservation depends on bottom water oxygenation of an ecosystem.

The broad spectrum of microscopic palynomorphs encompass mainly the allochthonous terrestrial debris from land plants, especially pollen and associated land derived fungal, bryophytic and pteridophytic spores. These can be washed out by water currents and wind into far reaching environments. The autochthonous pollen /spore are derived from hydrophytes, algae and associated fungal spores along with the organic fragments showing affinity with insects and annelid worms, microforaminifer linings etc. The study was taken up to unravel surface sediment stacking patterns and the accommodation space of organic matter type in the Mahanadi Delta as indicators of its depositional microenvironments. The study provides a modern analogue of palynology and palynofacies study in surface sediments responding to the present day geomorphology and external factors like climate and relative sea level in order to understand the palaeo-deltaic depositional environment.

Location:The deltaic plain of the Mahanadi river covers $0.9 \times 10^{4} \mathrm{sq} . \mathrm{km}$ and lies between $85^{\circ} 40^{\prime}: 86^{\circ} 45^{\prime} \mathrm{E}$ and $19^{\circ}$ $40^{\prime}: 20^{\circ} 35^{\prime} \mathrm{N}$. The climatic setting is tropical with hot and humid monsoonal climate. With an average annual rainfall of ca.1572 mm, over $70 \%$ is precipitated during the southwest monsoon between June to October. The 


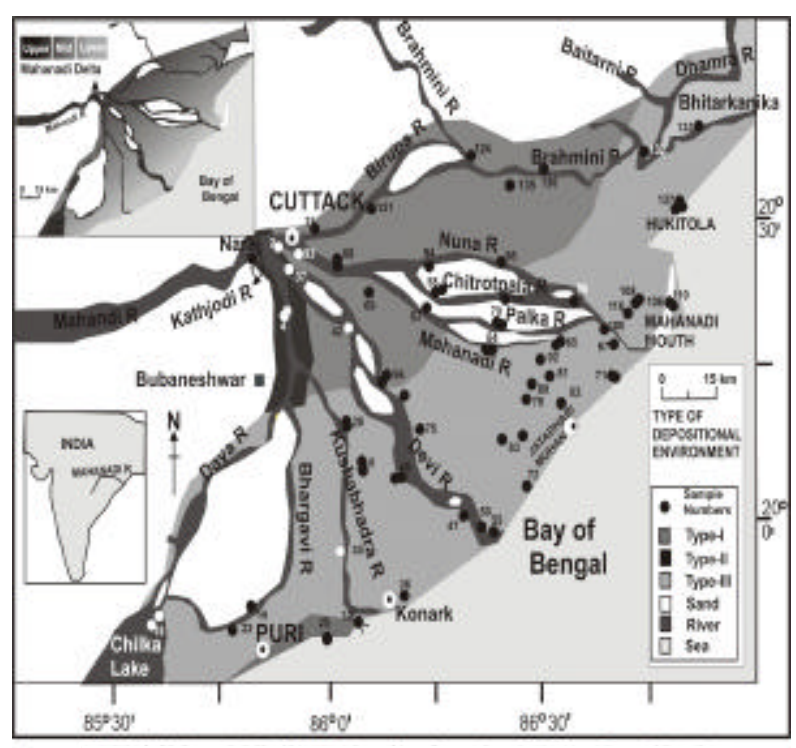

Fig.1. Mahanadi Delta system, location of samples and type of despositional environment (I-High BUD; II- POM, Pm; III-Low $O M)$.

Mahanadi river starts building up its delta plain from Naraj where the undivided Mahanadi branches forming its distributary system (Fig.1) ramifying in the delta plain area. Tectonics and Evolution: The Mahanadi River is an arcuate delta system formed in a tectonic downwarp of the Gondwana graben believed to be a failed arm of the triple junction on the Eastern Indian coast passive margin (Jagannathan etal, 1983). Subsurface sediments of the Mahanadi Basin range in age from Late Jurassic/Early Cretaceous to Recent. Sambasiva Rao etal. (1978) dated the Oldest beach ridge/ strandline in the Mahanadi deltaic area to be $\pm 6000 \mathrm{yrs}$. B.P. The major force of delta building seems to have spanned in the Late Holocene between 6000 yrs. B.P. to 800 yrs. B.P. (Mohanti, 1993). Shoreline has further shifted seaward during the last 800 years. Deltaic progradation continued in a northerly direction. Dyanamic processes: Fluvial and nearshore marine processes throw the most dynamic impact on the deltaic coast during the southwest monsoon. The undivided Mahanadi river at its delta head at Mundali/Naraj carries an annual discharge of water depending upon the amount of rainfall in the catchment area, a very high water discharge upto 45,000 cumsec may be flushed at the delta head during very high floods in southwest monsoonal period and flowing through the distributary system may ravage the low lying deltaic tract enormously. Mahanadi river deltaic coast is microtidal with a mean tidal range measuring $1.29 \mathrm{~m}$.Tidal cycle is semi-diurnal. It is principally a wave-dominated coast during the southwest monsoonal season, while during the non-monsoonal period it is mixed wave and tide dominated. The northeast monsoon (between December - early January) is much more milder in its dynamic activity.
Sediment deposition: Sediment deposition in the Mahanadi River delta is principally monsoon-dominated. The river channels surround the flood plains, sea waves, tidal creeks, tidal swales, spits, off shore bars and enclosed lagoons. Significant work on the morphology of this delta system has been carried out earlier (Sambasiva Rao et al.,1978; Meijerinck, 1982-83; and Nioyogi, 1975). At present Debi, Kuakhai and Birupa are active Mahanadi river distributory channels and meet the present coastline (Mahalik, 2006). Diffusion of effluents and sediment dispersion patterns at the river mouths have been discussed by Wright (1985). Because of the low volumetric capacity of the Mahanadi River and high flushing velocity, the suspended load during the monsoon enters into the Bay of Bengal probably as a hypopycnal buoyant plume and / or friction dominated plane jet depending on the volume of fluvial discharge and the hydrodynamic conditions of the innershelf (Ray and Mohanti, 1989). The turbid plume may extend ca.15 $\mathrm{km}$ or more into the Bay of Bengal during the monsoonal months of high fluvial discharge (Mohanti, 1993).

The fine suspended sediment get deposited during monsoons in the flood plain and marginal tidally influenced environments like grassy swamps mangrove swamps, tidal flats and also in the deeper offshore environment. Residual estuarine circulation in nonmonsoonal months also distributes fine sediments in the estuarine/deltaic environments (Meijerink, 1982-83). Greater amount of water discharge and sediment load are carried by the active channel of the Debi river distributary.

\section{MATERIALS AND METHODS}

Total 57 surface sediment samples were collected from Birupa River System, Kathjodi-Debi River system and Kuakhai River System comprising Upper, Middle and Lower Deltaic part of Mahanadi River as shown in Fig.1. and Tables 1 to 3 . Ten gram air dried soil sediment was treated with $10 \% \mathrm{KOH}$ for 5-10 min. on sand bath.

The filtrate (150 mesh size) was allowed to settle overnight. After draining out the supernatant, the sediment was treated with HF (Hydrofluoric acid) for two days or unless the silica was dissolved. HF was removed by centrifuging at $2000 \mathrm{rpm}$ for $10 \mathrm{~min}$. The acetolysis process was carried out using glacial acetic acid, anhydrous acetic acid and sulphuric acid following Erdtman, 1983. The processed sediment was filtered through 600 mesh size $(>15 \mu \mathrm{m})$ and the residue stored in $50 \%$ glycerine and glass distilled water (1:1). The final sediment solution was made up to $10 \mathrm{ml}$. One drop per slide was studied under light microscope (Olympus BX52). An average of 8-10 slides per sample was studied depending on the richness of organic debris in sample. The identification of organic matter was done following Tyson, 1993 and 1994; Taylor, et al., 1998. 


\section{RESULTS}

Three types of depositional environment were identified on the basis of the relative abundance of Particulate Organic Matter (POM). The Type-I constitute mainly Black Unidentified Debris (BUD) followed by moderate Amorphous Organic Matter (AOM) and Structured Organic Matter (SOM) indicating moderate fluvial energy and nearby provenance and oxic depositional conditions (Fig.2 and 3). Type II constitute moderate to high percentages of Palynomorphs (Pm) such as pollen, spores, algal cysts and fungal mycelia (Fig.4), SOMmainly plant cuticles and moderate BUD indicating low fluvial energy during most of the time and in situ deposition of organic matter under anoxic conditions. Type-III constitutes mainly Amorphous Organic Matter (AOM) with very low POM mainly derived from in situ algal matter or transported from nearby provenance indicating oxic to dysoxic depositional conditions. The Mahanadi River delta has been here divided into upper, middle and lower delta plain (UDP, MDP and LDP) during the description below.

Koyakhai river: At the apex of the Koyakhai distributary the dominance of MOA by 80 per cent and low BUD represents the Type III depositional environment in the channel sediments (Figs.2 and 5) deposited under oxic/ dysoxic conditions. However, the flood plain sediment at the apex of the Daya tributary in the UDP shows high BUD (Table-1). The Kushbhadra, Bhargavi and the Daya distributary river channels constituting the MDP and LDP is comparatively rich in palynomorphs, moderate MOA, structured aquatic plant debris and BUD representing Type-II anoxic depositional environment. The foreshore sample near Puri (south-eastern part) shows Type-III depositional environment which is rich in MOA. However, further northward near the Konark in the estuarine sediment the Type-II depositional environment was observed. The sediment here is rich in mangrove and other estuarine palynomorphs such as Avicennia (2\%), Rhizophora (4\%), Sonneratia (1\%), Excoecaria (2\%), Clerodendron-Justicia- Casuarina- Euphorbiaceae (2\%) and moderate BUD and SOM. Towards the LDP and UDP only the Kushbhadra river channel shows comparatively high BUD i.e., Type -I depositional environment. Thus, the MDP and the estuarine sediment in LDP show high rate of POM preservation. Out of three distributary channels (Daya, Bhargavi and Kushbhadra) of Koyakhai river system only Kushbhadra shows fluvial depositional environment whereby, abundance of oxidized POM indicated by BUD is observed in UDP as well as in LDP. Thus, only Kushbhadra is an active channel in the Koyakhai river system.

Debi river: The UDP of the Debi distributary channel shows dominance of Type III oxic/dysoxic depositional environment at five locations in 17 sediment samples

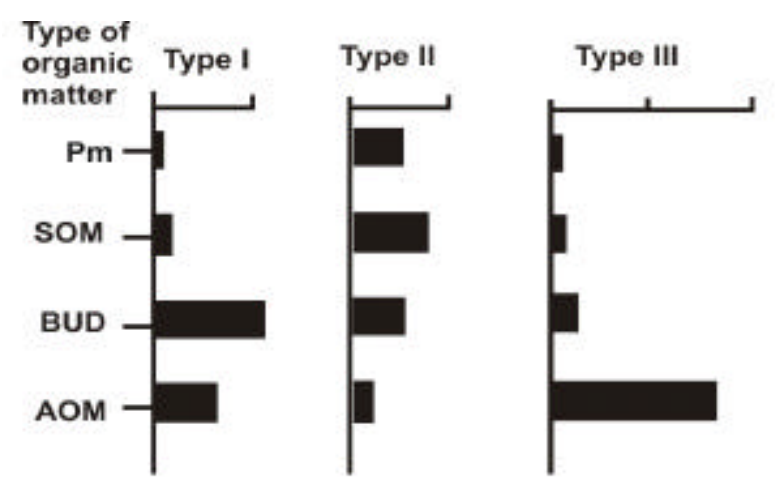

Fig.2. Percentage of Particulate Organic matter (POM) in surface sediment, Mahanadi River Delta.

(Table-1 contd.). The river banks and flood plains however, reveal preservation of POM. The MDP and LDP, however, shows Type-II indicating low fluvial energy allowing POM stacking in the sediment. The Debi river mouth in the LDP shows sea water intrusion creating saline bottom and allowing POM to settle down and get preserved under anoxic depositional environment.

Kathjodi- Mahanadi and Palka River: This distributary channel shows abundance of AOM characterized by Type- III depositional environment in channel sediment and palaeobeach (Table-1 contd.). However, the flood plains in the UDP show POM preservation representing Type-II depositional environment. In LDP near Jatadhari Muhan the depositional environment is Type-II that indicates stacking of POM in this part of the delta under anoxic conditions.

Chitrotpala, Nuna and Birupa river System: The upper part of the delta is high in AOM representing Type- III oxic-dysoxic depositional environment (Table-1 contd.). The MDP and UDP are high in POM representing TypeII depositional environment. The mouths of Mahanadi, Nuna and Jambu nadi including Hukitola spit again show Type-II anoxic depositional environment both in channel sediments as well as in spit area. This indicates low fluvial energy and is protected from direct sea water influence during most of the time in the year.

Birupa River System: The channel sediments from UDP show high AOM thus representing Type-III oxic/dysoxic depositional environment. Similar environment was recorded at the confluence zone of Brahmini River in the UDP. The MDP and LDP however show high POM preservation representing Type-II anoxic depositional environment.

Depositional Environment in Mahanadi River System Type $\mathrm{I}$ : $(\mathrm{BUD}>\mathrm{AOM}>\mathrm{SOM}>\mathrm{Pm})$

Black indeterminate phytoclasts categorised as BUD are of heterogonous nature. Most of these are lathe shaped and show angular margins indicating a short distance transport. The Amorphous organic matter is scattered type and show heterogonous assemblage. These are 


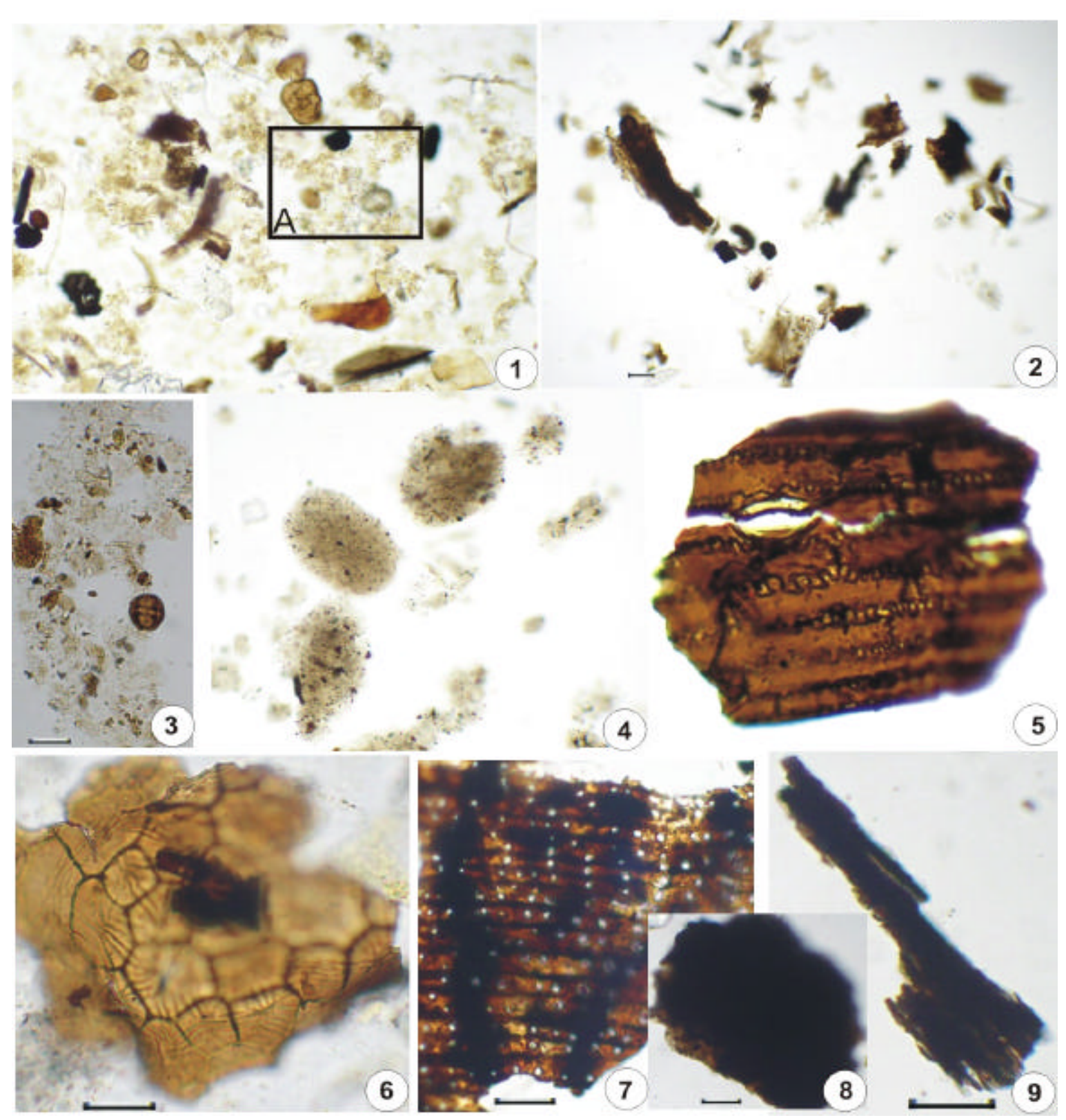

Fig. 3. 1. Pm- POM such as pollen, spores, faint features of Spirogyra filaments (A) 2. Unstructured black coaly debris; 3Rhizophora pollen and amorphous organic matter common in LDP; 4- Rounded amorphous organic matter in LDP; 5 and 6Structured Organic Matter - plant cuticles. 7- Structured Woody Remains, trachieds; 8- NSC Non-Structured Charcoal; 9- Poorly Preserved Woody Remains.

colourless showing fine faded lamellae like structures with dark black unidentifiable inclusions arranged into linear spiral structure perhaps representing the Spirogyra mass and pyrenoids along with the fine BUD inclusions (Fig. 3). Only few land derived pollen/spores were recorded. This type of depositional environment was recorded at four sites dominating in the Koyakhai river system (Fig.1). The lathe shaped coaly debris are lighter in weight transported from land or adjacent flood area. This suggests that the Koyakhai- Kushbhadra river channel is active allowing the flow of POM which is enhanced during rainy season. Dominance of Spirogyra mass here indicates the influx of nitrogen and phosphorous from nearby agricultural land. Thus it is inferred that the nutrient input in the apex of the delta is transported through Daya river feeding the Chilka lake (Table 1). The sediments are preserved here under oxic to dysoxic conditions.

Type II: $(\mathrm{Pm}>\mathrm{SOM}>\mathrm{BUD}>\mathrm{AOM})$

This type dominates in the MDP and LDP of all the distributary channels. The sediment is rich in variety of terrestrial derived organic matter (Fig. 4). Identifiable 


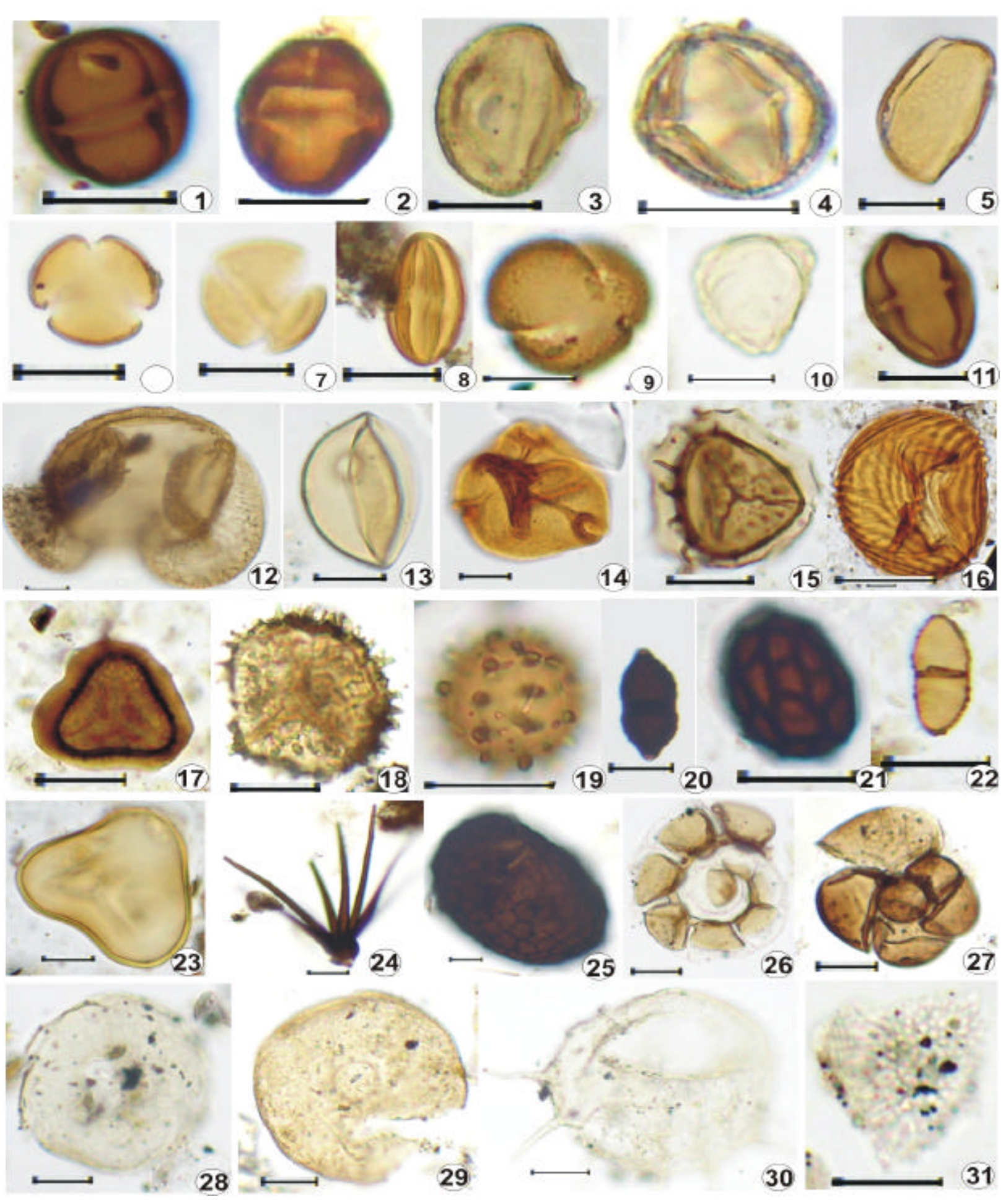

Fig. 4. 1 and 2-Rhizophora; 3-Sonneratia; 4-Excoecaria; 5-Liliaceae; 6-Fabaceae; 7-Lamiaceae; 8-Strobilanthes; 9-Euphorbiaceae; 10-Casuarina; 11- Anacardiaceae; 12- Pinus; 13-Cerealia; 14-18- Trilete Cryptogamic spores; 19- algal cyst; 20-21-fungal spores;22-Ascopore; 23-Acrostichum; 24- tetraploae; 25-Fungal spore; 26 \&27 Estuarine Foraminifera lining; *28-Arcellacean -Trigonopyxis arcula; *29-Arcella artocrea; *30-Centropyxis spinosa; *31-Loricated Tintinnopsis. *Zooplanktons. All scale = $10 \mu \mathrm{m}$ 
Table 1. Mahanadi Delta - Koyakhai River System

\begin{tabular}{|c|c|c|c|c|c|c|}
\hline S.No & Location & $\begin{array}{l}\text { Latitude } \\
\text { Longitude }\end{array}$ & $\begin{array}{l}\text { Name of the } \\
\text { River }\end{array}$ & Microenvironment & Sample No's & $\begin{array}{l}\text { Type of } \\
\text { Depositional } \\
\text { Environment }\end{array}$ \\
\hline 1 & Sanamundali & $\begin{array}{l}\text { N } 20^{0} 26.990^{1} \\
\text { E } 85^{0} 44.861^{1}\end{array}$ & Mahanadi River & Channel Sediment & 1-MR/CS/1 & VII \\
\hline 2 & & $\begin{array}{l}\text { N } 20^{0} 28.989^{\prime} \\
\text { E } 85^{0} 46.127^{\prime}\end{array}$ & $\begin{array}{l}\text { Apex of the } \\
\text { Mahanadi Delta }\end{array}$ & Channel Sediment & 2-AMD/CS1/3 & VII \\
\hline 4 & Kalyanpur & $\begin{array}{l}\text { N } 20^{0} 22.606^{1} \\
\text { E } 85^{0} 51.929^{1}\end{array}$ & $\begin{array}{l}\text { Koyakhai River } \\
\text { System }\end{array}$ & $\begin{array}{l}\text { Channel Sediment } \\
\text { Point Bar } \\
\text { Channel Bar } \\
\text { Flood Plain }\end{array}$ & $\begin{array}{l}4-\mathrm{KK} / \mathrm{CS} / 14 \\
4-\mathrm{KK} / \mathrm{PB} / 15 \\
4-\mathrm{KK} / \mathrm{CB} / 16 \\
4-\mathrm{KK} / \mathrm{FP} / 17\end{array}$ & $\begin{array}{l}\text { VII } \\
\text { VII } \\
\text { VII } \\
\text { I }\end{array}$ \\
\hline 8 & Kantapada & $\begin{array}{l}\text { N } 20^{0} 13.07^{1} \\
\text { E } 86^{0} .00 .07^{1}\end{array}$ & $\begin{array}{l}\text { Prachi(Paleochann } \\
\text { el) }\end{array}$ & $\begin{array}{l}\text { Channel sediment } \\
\text { Flood plain } \\
\text { River Dune }\end{array}$ & $\begin{array}{l}8-\mathrm{PN} / \mathrm{CS} / 29 \\
8-\mathrm{PN} / \mathrm{FP} / 30 \\
8-\mathrm{PN} / \mathrm{RD} / 31\end{array}$ & $\begin{array}{l}\text { VII } \\
\text { IV } \\
\text { VII }\end{array}$ \\
\hline 9 & Lingpur & $\begin{array}{l}\text { N } 20^{0} 12.559^{1} \\
\text { E } 85^{0} 51.181^{1}\end{array}$ & Daya River & $\begin{array}{l}\text { Channel Sediment } \\
\text { Sediment Bar } \\
\text { Flood Plain }\end{array}$ & $\begin{array}{l}\text { 9-DR/CS/32 } \\
\text { 9-DR/SB/33 } \\
\text { 9-DR/FP/34 }\end{array}$ & $\begin{array}{l}\text { VII } \\
\text { VII } \\
\text { VII }\end{array}$ \\
\hline 15 & & $\begin{array}{l}\mathrm{N} 19^{0} 51.433^{1} \\
\mathrm{E} 85^{0} 32.602^{1} \\
\mathrm{~N} 19^{0} 51.676^{1} \\
\mathrm{E} 85^{0} 32.922^{1}\end{array}$ & Chilika Lake & $\begin{array}{l}\text { Mud Bank } \\
\text { Centre }\end{array}$ & $\begin{array}{l}\text { 15-CL/Mud/46 } \\
\text { 15-CL/CS1/47 } \\
15-C L / C S 2 / 48\end{array}$ & $\begin{array}{l}\text { VI } \\
\text { VI } \\
\text { II }\end{array}$ \\
\hline 19 & Chandanpur & $\begin{array}{l}\mathrm{N} 19^{0} 53.459^{1} \\
\mathrm{E} 85^{0} 49.76^{1}\end{array}$ & Bhargavi River & Channel Sediment & 19-BR/CS/63 & I \\
\hline 22 & Mangrajpur & $\begin{array}{l}\text { N } 19^{0} 50.126^{1} \\
\text { E } 85^{0} 49.133^{1}\end{array}$ & Samang Lake & 1 Sediment & 22-SL/1/66 & IV \\
\hline 25 & Baleswar & $\begin{array}{l}\mathrm{N} 19^{0} 49.651^{1} \\
\mathrm{E} 85^{0} 56.642^{1}\end{array}$ & Beach & Foreshore & $25-\mathrm{B} / \mathrm{FS} / 78$ & VII* \\
\hline 28 & Banamalipur & $\begin{array}{l}\text { N } 20^{0} 10.487^{\prime} \\
\text { E } 86^{0} 00.078^{1}\end{array}$ & Kushabhadra River & $\begin{array}{l}\text { Channel Sediment } \\
\text { Point Bar } \\
\text { Natural Levee } \\
\text { Flood Plain }\end{array}$ & $\begin{array}{l}28-\mathrm{KR} / \mathrm{CS} / 88 \\
28-\mathrm{KR} / \mathrm{PB} / 89 \\
28-\mathrm{KR} / \mathrm{NL} / 90 \\
28-\mathrm{KR} / \mathrm{FP} / 91\end{array}$ & $\begin{array}{l}\text { II } \\
\text { I } \\
\text { II } \\
\text { II }\end{array}$ \\
\hline 30 & Gop & $\begin{array}{l}\text { N } 19^{0} 59.044^{1} \\
\text { E } 86^{0} 00.784^{1}\end{array}$ & Kushabhadra River & $\begin{array}{l}\text { Channel Sediment } \\
\text { Point Bar } \\
\text { Natural Levee } \\
\text { Flood Plain }\end{array}$ & $\begin{array}{l}30-\mathrm{KR} / \mathrm{CS} / 96 \\
30-\mathrm{KR} / \mathrm{PB} / 97 \\
30-\mathrm{KR} / \mathrm{NL} / 98 \\
30-\mathrm{KR} / \mathrm{FP} / 99\end{array}$ & $\begin{array}{l}\text { I } \\
\text { I } \\
\text { I } \\
\text { I }\end{array}$ \\
\hline 32 & $\begin{array}{l}\text { Ramachandi } \\
\text { (Mouth 1) }\end{array}$ & $\begin{array}{l}\mathrm{N} 19^{0} 51.261^{1} \\
\mathrm{E} 86^{0} 03.620^{1}\end{array}$ & Kushabhadra River & $\begin{array}{l}\text { Estuary } \\
\text { Sediment-1 }\end{array}$ & $32-\mathrm{KR} / \mathrm{E}(\mathrm{M} 2) / 1 / 106$ & III \\
\hline 36 & Chandrabagh & $\begin{array}{l}\text { N } 19^{0} 51.936^{1} \\
\text { E } 86^{0} 06.814^{1}\end{array}$ & Konark Beach & Foreshore & $36-\mathrm{B} / \mathrm{FS} / 122$ & VI \\
\hline
\end{tabular}

cellular phytoclasts, and dark brown partially oxidised leaf cuticular tissue indicate allochthonous organic matter. The angularity of debris suggests its nearby source from the flood plain. Pollen assemblage is moderate, may be because of open fallow land having less vegetation nearby. Pollen belong to Cassia, Clerodendron, Acanthus, Strobilanthes and Bisaccate wind drifted Pinus pollen. Fungal mycelia, and spores that are saprophytic on terrestrial plants are common. Estuarine micro foraminfera linings and dominance of insect egg shells, algal cysts and cysts of unknown affinity were observed (Fig.4). Microscopic arthropods and their egg shells dominate these sediments. Presence of VAM
(Vesicular Arbuscular Mycorrhyiza) fungi that are found in soil associated with plants of weak root system indicate the on going process of vegetational stability within the MDP and LDP ecosystem. Scatterred and fibrous type of amorphous organic matter are light brown to colourless. The pyrenoids are arranged in zig-zag fashion showing lineage to fresh water algae ie., Spirogyra (Fig. 3). Abundance of Spirogyra indicates high autotrophic level in ecosystem which derives nutrients from adjacent agricultural land. During summers, the decay of these perhaps increased biological and chemical oxidation demand resulting into anoxic water column allowing stacking of organic matter under anoxic bottom. In the 
Table 1. Contd. : Mahanadi And Chitrotpala-Kathjodi-Debi River Systems

\begin{tabular}{|c|c|c|c|c|c|c|c|}
\hline S N. & Location & $\begin{array}{l}\text { Latitude } \\
\text { Longitude }\end{array}$ & $\begin{array}{l}\text { Name of the } \\
\text { River }\end{array}$ & $\begin{array}{c}\text { Microenvi } \\
\text { ronment }\end{array}$ & $\begin{array}{l}\text { Sample No. } \\
\text { in Fig.1 }\end{array}$ & $\begin{array}{l}\text { No. Sample } \\
\text { per location }\end{array}$ & $\begin{array}{l}\text { Type of } \\
\text { Depositional } \\
\text { Environment }\end{array}$ \\
\hline \multirow[t]{3}{*}{37} & Khapuria & $\mathrm{N} 20^{0} 26.092^{1}$ & Katjuri River & $\mathrm{CS}$ & 37 & 124 & III \\
\hline & & E $85^{0} 54.029^{1}$ & & $\mathrm{~PB}$ & 37 & 125 & III \\
\hline & & & & SB & 37 & 126 & III \\
\hline \multirow[t]{3}{*}{42} & Ukundara & $\mathrm{N} 20^{\circ} 18.528^{1}$ & Devi River & $\mathrm{CS}$ & 42 & 148 & III \\
\hline & & E $86^{0} 01.987^{1}$ & & PB & 42 & 149 & III \\
\hline & & & & FP & 42 & 150 & II \\
\hline \multirow[t]{4}{*}{43} & Alipingala & $\mathrm{N} 20^{0} 13.093^{1}$ & Devi River & CS & 43 & 151 & III \\
\hline & & E $86^{\circ} 08.842^{1}$ & & SB & 43 & 263 & III \\
\hline & & & & RB & 43 & 152 & II \\
\hline & & & & FP & 43 & 153 & II \\
\hline \multirow[t]{4}{*}{45} & Balipadha & $\mathrm{N} 20^{\circ} 04.763^{1}$ & Devi River & CS & 45 & 157 & II \\
\hline & & E $86^{\circ} 10.243^{1}$ & & PB & 45 & 158 & III \\
\hline & & & & FP & 45 & 159 & III \\
\hline & & & & PR & 45 & 160 & III \\
\hline 47 & & $\mathrm{~N} 20^{\circ} 01.829^{1}$ & Devi River & $\mathrm{CS}$ & 47 & 171 & II \\
\hline 50 & & $\begin{array}{l}\text { E } 86^{0} 19.646^{1} \\
\text { N } 19^{0} 59.826^{1} \\
\text { E } 86^{0} 20.414^{1}\end{array}$ & Devi River & CS & 50 & 175 & II \\
\hline 55 & & $\begin{array}{l}\text { N } 19^{0} 58.282^{1} \\
\text { E } 86^{0} 21.755^{1}\end{array}$ & Devi River & $\mathrm{CS}$ & 55 & 180 & II \\
\hline 56 & Jaswantpur & $\begin{array}{l}\text { N } 20^{\circ} 13.652^{1} \\
\text { E } 86^{0} 09.483^{1}\end{array}$ & Gobari Nadi & $\mathrm{CS}$ & 56 & 181 & III \\
\hline \multirow[t]{3}{*}{58} & Uttarkula & N $20^{\circ} 23.341^{1}$ & Chitrotpala River & $\mathrm{CS}$ & 58 & 185 & III \\
\hline & & E $86^{0} 13.642^{1}$ & & $\mathrm{CB}$ & 58 & 186 & I \\
\hline & & & & SB & 58 & 187 & III \\
\hline \multirow[t]{3}{*}{59} & Kolabuda & $\mathrm{N} 20^{\circ} 24.329^{1}$ & Chitrotpala River & $\mathrm{CS}$ & 59 & 188 & III \\
\hline & & E $86^{0} 21.066^{1}$ & & SB & 59 & 189 & III \\
\hline & & & & FP & 59 & 190 & II \\
\hline \multirow[t]{4}{*}{60} & Aitpur & $\mathrm{N} 20^{0} 26.065^{1}$ & Mahanadi River & CS & 60 & 191 & III \\
\hline & & E $86^{0} 00.871^{1}$ & & $\mathrm{CB}$ & 60 & 192 & III \\
\hline & & & & PB & 60 & 193 & III \\
\hline & & & & FP & 60 & 194 & II \\
\hline \multirow[t]{3}{*}{63} & Tarapur & $\mathrm{N} 20^{\circ} 21.701^{1}$ & Mahanadi River & $\mathrm{CS}$ & 63 & 206 & III \\
\hline & & E $86^{0} 13.692^{1}$ & & SB & 63 & 207 & III \\
\hline & & & & FP & 63 & 208 & III \\
\hline \multirow{4}{*}{64} & Tirttol & $\mathrm{N} 20^{\circ} 18.984^{\circ}$ & Mahanadi River & CS & 64 & 209 & III \\
\hline & & E $86^{0} 22.485^{1}$ & & $\mathrm{CB}$ & 64 & 210 & III \\
\hline & & & & SSB & 64 & 211 & III \\
\hline & & & & FP & 64 & 212 & II \\
\hline \multirow[t]{2}{*}{65} & Kujang & $\mathrm{N} 20^{\circ} 19.629^{\prime}$ & MR \& PR & CS & 65 & 214 & II \\
\hline & & E $86^{0} 32.179^{1}$ & & FP & 65 & 215 & II \\
\hline \multirow[t]{2}{*}{67} & Paradeep & N $20^{\circ} 22.302^{1}$ & Mahanadi River & CS & 67 & 218 & III \\
\hline & $\begin{array}{l}\text { (Nehru } \\
\text { Bungalow) }\end{array}$ & E $86^{0} 09.639^{1}$ & & & & & \\
\hline \multirow[t]{2}{*}{69} & Somapur & N $20^{\circ} 22.990^{1}$ & Paika Nadi & $\mathrm{CS}$ & 69 & 221 & II \\
\hline & & E $86^{\circ} 04.375^{1}$ & & & & & \\
\hline \multirow[t]{4}{*}{70} & Kolar & N $20^{\circ} 20.585^{1}$ & Paika River & CS & 70 & 222 & III \\
\hline & & E $86^{0} 22.946^{1}$ & & $\mathrm{CB}$ & 70 & 223 & III \\
\hline & & & & SB & 70 & 224 & III \\
\hline & & & & $\mathrm{CI}$ & 70 & 225 & II \\
\hline 71 & Paradeep & $\begin{array}{l}\text { N } 20^{0} 15.091^{1} \\
\text { E } 86^{0} 39.352^{1}\end{array}$ & Beach & IS & 71 & 226 & III \\
\hline 72 & & $\begin{array}{l}\text { N } 20^{\circ} 04.562^{1} \\
\text { E } 86^{\circ} 27.267^{1}\end{array}$ & Beach & IS & 72 & 231 & III \\
\hline 75 & & $\mathrm{~N} 20^{\circ} 09.638^{1}$ & Alaka Nadi & CS & 75 & 238 & II \\
\hline 77 & Garia & $\begin{array}{l}\text { E } 86^{0} 11.876^{1} \\
\text { N } 20^{\circ} 05.175^{1} \\
\text { E } 86^{0} 25.694^{1}\end{array}$ & Sankha Nadi & CS & 77 & 240 & II \\
\hline 79 & Japabhuan & $\begin{array}{l}\text { N } 20^{0} 12.281^{1} \\
\text { E } 86^{0} 25.533^{1}\end{array}$ & Tigaria Nadi & $\mathrm{CS}$ & 79 & 242 & II \\
\hline 80 & Irrebina & $\begin{array}{l}\text { N } 20^{\circ} 13.207^{1} \\
\text { E } 86^{0} 26.570^{1}\end{array}$ & Basuli Nadi & $\mathrm{CS}$ & 80 & 243 & II \\
\hline 81 & Balituja & $\begin{array}{l}\text { N } 20^{\circ} 14.258^{1} \\
\text { E } 86^{\circ} 31.739^{1}\end{array}$ & Mahang Nadi & $\mathrm{CS}$ & 81 & 244 & II \\
\hline 82 & & $\begin{array}{l}\text { N } 20^{\circ} 11.300^{1} \\
\text { E } 86^{0} 31.106^{1}\end{array}$ & $\begin{array}{l}\text { Ancient Beach } \\
\text { Ridge }\end{array}$ & $\mathrm{ABR}$ & 82 & 247 & IV \\
\hline 83 & Goda & $\begin{array}{l}\text { N } 20^{0} 06.120^{1} \\
\text { E } 86^{0} 23.009^{1}\end{array}$ & & PBR & 83 & 249 & II \\
\hline 92 & Samatrapur & $\begin{array}{l}\text { N } 20^{0} 15.994^{1} \\
\text { E } 86^{0} 30.034^{1}\end{array}$ & $\begin{array}{l}\text { Ancient } \\
\text { Ridge }\end{array}$ & $\mathrm{ABR}$ & 92 & 262 & II \\
\hline
\end{tabular}


Table 1. contd. Birupa river system.

\begin{tabular}{|c|c|c|c|c|c|c|c|}
\hline S.No & Location & Lat-Long & $\begin{array}{l}\text { Name of the } \\
\text { River }\end{array}$ & $\begin{array}{l}\text { Microenviro } \\
\text { nment }\end{array}$ & $\begin{array}{l}\text { Sample No. } \\
\text { in Fig.1 }\end{array}$ & $\begin{array}{l}\text { No. sample/ } \\
\text { location }\end{array}$ & $\begin{array}{l}\text { Type of } \\
\text { Depositional } \\
\text { Environment }\end{array}$ \\
\hline 93 & $\begin{array}{l}\text { Mahanadi Vihar } \\
\text { Cuttack }\end{array}$ & $\begin{array}{l}\text { N } 20^{0} 28^{1} 16.7^{11} \\
\text { E } 85^{0} 54^{1} 44.2^{11}\end{array}$ & Mahanadi.R & $\mathrm{CS}$ & 93 & 264 & III \\
\hline 94 & Kandasahi & $\begin{array}{l}\text { N } 20^{0} 27^{1} 00.5^{11} \\
\text { E } 86^{0} 11^{1} 56.6^{11}\end{array}$ & Nuna.R & $\begin{array}{l}\text { CS } \\
\text { SB }\end{array}$ & $\begin{array}{l}94 \\
94\end{array}$ & $\begin{array}{l}266 \\
267\end{array}$ & $\begin{array}{l}\text { III } \\
\text { III }\end{array}$ \\
\hline 96 & Kalaparha & $\begin{array}{l}\text { N } 20^{\circ} 27^{\prime} 07.1^{11} \\
\text { E } 86^{0} 25^{1} 16.1^{11}\end{array}$ & Nuna.R & $\begin{array}{l}\mathrm{CS} \\
\mathrm{SB}\end{array}$ & $\begin{array}{l}96 \\
96\end{array}$ & $\begin{array}{l}276 \\
277\end{array}$ & $\begin{array}{l}\text { III } \\
\text { III }\end{array}$ \\
\hline 98 & Baulpara & $\begin{array}{l}\text { N } 20^{0} 24^{1} 16.8^{11} \\
\text { E } 86^{0} 34^{1} 38.7^{11}\end{array}$ & Nuna.R & CS & 98 & 282 & II \\
\hline 100 & Mahakud & $\begin{array}{l}\text { N } 20^{0} 19^{1} 54.0^{11} \\
\text { E } 86^{0} 39^{1} 48.5^{11}\end{array}$ & Mahanadi.R & $\mathrm{CS}$ & 100 & 285 & III \\
\hline 106 & Jambu Lock & $\begin{array}{l}\text { N } 20^{0} 23^{1} 45.4^{11} \\
\text { E } 86^{0} 43^{1} 30.5^{11}\end{array}$ & Jambu Nadi & MS & 106 & 291 & II \\
\hline 108 & Hukitola Bay & $\begin{array}{l}\text { N } 20^{\circ} 24^{1} 29.6^{11} \\
\text { E } 86^{0} 45^{1} 55.2^{11}\end{array}$ & Hukitola Bay & CS & 108 & 293 & II \\
\hline 110 & Hukitola port & $\begin{array}{l}\text { N } 20^{0} 24^{1} 17.3^{11} \\
\text { E } 86^{0} 47^{1} 17.4^{11}\end{array}$ & Hukitola spit & $\begin{array}{l}\text { CS } \\
\text { Spit }\end{array}$ & $\begin{array}{l}110 \\
110\end{array}$ & $\begin{array}{l}295 \\
297\end{array}$ & $\begin{array}{l}\text { II } \\
\text { II }\end{array}$ \\
\hline 116 & Ramnagar & $\begin{array}{l}\text { N } 20^{0} 21^{1} 35.8^{11} \\
\text { E } 86^{0} 40^{1} 32.8^{11}\end{array}$ & $\begin{array}{l}\text { Paleo Beach } \\
\text { Ridge }\end{array}$ & PBR & 116 & 303 & III \\
\hline 119 & Banika & $\begin{array}{l}\text { N } 20^{0} 30^{1} 48.1^{11} \\
\text { E } 86^{0} 55^{1} 57.4^{11}\end{array}$ & Birupa.R & CS & 119 & 308 & III \\
\hline 121 & Jasrajpur & $\begin{array}{l}\text { N } 20^{0} 35^{1} 27.6^{11} \\
\text { E } 86^{0} 05^{1} 45.8^{11}\end{array}$ & Birupa.R & $\mathrm{CS}$ & 121 & 312 & III \\
\hline 124 & $\begin{array}{l}\text { Khargpur } \\
\text { (before } 1 \mathrm{~km} \text { ) }\end{array}$ & $\begin{array}{l}\text { N } 20^{0} 38^{1} 05.2^{11} \\
\text { E } 86^{0} 18^{1} 17.3^{11}\end{array}$ & Birupa.R & CS & 124 & 316 & III \\
\hline 130 & Chandanpur & $\begin{array}{l}\text { N } 20^{0} 36^{1} 38.2^{11} \\
\text { E } 86^{0} 29^{1} 21.3^{11}\end{array}$ & Brahmani.R & $\mathrm{CS}$ & 130 & 328 & III \\
\hline 132 & Rajgath & $\begin{array}{l}\text { N } 20^{\circ} 36^{1} 15.3^{11} \\
\text { E } 86^{0} 44^{1} 03.4^{11}\end{array}$ & Brahmani.R & $\mathrm{FP}$ & 132 & 335 & II \\
\hline 133 & Gupti & $\begin{array}{l}\text { N } 20^{0} 39^{1} 15.4^{11} \\
\text { E } 86^{0} 51^{1} 03.6^{11}\end{array}$ & Maipura.N & $\mathrm{CS}$ & 133 & 336 & II \\
\hline 135 & Kanara & $\begin{array}{l}\text { N } 20^{0} 34^{1} 40.0^{11} \\
\text { E } 86^{0} 25^{1} 57.4^{11}\end{array}$ & Mud & Mud & 135 & 338 & II \\
\hline 137 & Penth & $\begin{array}{l}\text { N } 20^{0} 32^{1} 23.3^{11} \\
\text { E } 86^{0} 47^{\prime} 22.1^{11}\end{array}$ & Beach & $\begin{array}{l}\text { IS } \\
\mathrm{BC}\end{array}$ & $\begin{array}{l}137 \\
137 \\
\end{array}$ & $\begin{array}{l}341 \\
346 \\
\end{array}$ & $\begin{array}{l}\text { III } \\
\text { II }\end{array}$ \\
\hline
\end{tabular}

CS: Channel Sediment ; RB: River Bank; SB: Sediment Bar; PR: Paleo Ridge; SSB: Stabilized Sand Bar; RB: River Bank; CB: Channel Bar; IS: Inshore; PB: Point Bar; CI: Channel Island; FP: Flood Plain; ABR: Ancient Beach Ridge; PBR: Paleo Beach Ridge; RD: River Dune; MR: Mahanadi River; MS: Mangrove swamp; BC: Beach Clay; PBR: Paleo Beach Ridge; FS: Foreshore

LDP, the sediment represents typical estuarine environment (Fig.2). Pollen taxa recorded here belong to Rhizophoraceae, Avicennia, Sonneratia, Excoecaria, Lumnitzera, Casuarina. Soil fungi, and other terrestrial saprophytic fungal spores are common. Abundance of Trilete cryptogamic spores (Fig.4) were observed along with the mangrove pollen suggesting conducive humid environment. Fresh water Ammonia forams along with few estuarine foraminifers were recorded indicating brackish water-fresh water bottom. Characteristic features of Spirogyra filament are faintly evident in the amorphous organic matter (Fig.4.3) in the lower deltaic part of the Mahanadi river system and therefore it is inferred that these are transported from the upper deltaic part where the algal growth is in abundance. Amorphous organic matter show fine homogenous black inclusions and have round margins indicating its transport from long distance (Fig.3).
Type III (AOM> BUD->SOM> Pm)

The sediment mainly comprises of amorphous organic matter (Fig.3). In the UDP these are either in clumps of rounded shape or scattered morphology. Gradually towards the MDP and LDP the AOM attains rounded shape with pyrenoid inclusions in the form of small black dots as observed under light microscope. These debris have homogenous BUD inclusions except few show heterogeneity. Presence of Arcella artocrea and Trigonopyxis arcula (Fig.4), an Arcellacean generally found in drier ecosystem is common in MDP.

The Kathjodi and Debi River system comprising the central part of the Mahanadi delta complex shows abundance of algal growth particularly Spirogyra throughout in the channel except at few places (Table -2) indicating its slow, shallow and perennial discharge towards the sea. Presence of Tardigrad eggs, a 
Table 1. Mahanadi Delta - Koyakhai River System

\begin{tabular}{|c|c|c|c|c|c|c|c|}
\hline S.No & Location & $\begin{array}{l}\text { Latitude } \\
\text { Longitude }\end{array}$ & $\begin{array}{l}\text { Name of the } \\
\text { River }\end{array}$ & Microenvironment & $\begin{array}{l}\text { Sample } \\
\text { No. } \\
\text { location } \\
\text { Fig.1 } \\
\end{array}$ & $\begin{array}{l}\text { No. sample } \\
\text { per } \\
\text { location }\end{array}$ & $\begin{array}{l}\text { Type of } \\
\text { Depositional } \\
\text { Environment }\end{array}$ \\
\hline$\overline{1}$ & Sanamundali & $\begin{array}{l}\text { N } 20^{0} 26.990^{1} \\
\text { E } 85^{0} 44.861^{1}\end{array}$ & Mahanadi River & $\mathrm{CS}$ & 1 & 1 & III \\
\hline 2 & Naraj & $\begin{array}{l}\text { N } 20^{0} 28.989^{1} \\
\text { E } 85^{0} 46.127^{\prime}\end{array}$ & $\begin{array}{l}\text { Apex of the } \\
\text { Mahanadi Delta }\end{array}$ & $\mathrm{CS}$ & 2 & 3 & III \\
\hline 4 & Kalyanpur & $\begin{array}{l}\mathrm{N} 20^{0} 22.606^{1} \\
\mathrm{E} 85^{0} 51.929^{1}\end{array}$ & $\begin{array}{l}\text { Koyakhai River } \\
\text { System }\end{array}$ & $\begin{array}{l}\mathrm{CS} \\
\mathrm{PB} \\
\mathrm{CB} \\
\mathrm{FP}\end{array}$ & 4 & $\begin{array}{l}14 \\
15 \\
16 \\
17\end{array}$ & $\begin{array}{l}\text { III } \\
\text { III } \\
\text { III } \\
\text { I }\end{array}$ \\
\hline 8 & Kantapada & $\begin{array}{l}\text { N } 20^{0} 13.07^{1} \\
\text { E } 86^{0} .00 .07^{1}\end{array}$ & $\begin{array}{l}\text { Prachi } \\
\text { (Paleochannel) }\end{array}$ & $\begin{array}{l}\mathrm{CS} \\
\mathrm{FP} \\
\mathrm{RD}\end{array}$ & $\begin{array}{l}8 \\
8 \\
8\end{array}$ & $\begin{array}{l}29 \\
30 \\
31\end{array}$ & $\begin{array}{l}\text { III } \\
\text { III } \\
\text { III }\end{array}$ \\
\hline 9 & Lingpur & $\begin{array}{l}\text { N } 20^{0} 12.559^{1} \\
\text { E } 85^{0} 51.181^{1}\end{array}$ & Daya River & $\begin{array}{l}\mathrm{CS} \\
\mathrm{SB} \\
\mathrm{FP}\end{array}$ & $\begin{array}{l}9 \\
9 \\
9\end{array}$ & $\begin{array}{l}32 \\
33 \\
34\end{array}$ & $\begin{array}{l}\text { III } \\
\text { III } \\
\text { III }\end{array}$ \\
\hline 15 & & $\begin{array}{l}\text { N } 19^{0} 51.433^{1} \\
\text { E } 85^{0} 32.602^{1} \\
N 19^{0} 51.676^{1} \\
\text { E } 85^{0} 32.922^{1}\end{array}$ & Chilka Lake & $\begin{array}{l}\text { Mud } \\
\text { Centre }\end{array}$ & $\begin{array}{l}15 \\
15 \\
15\end{array}$ & $\begin{array}{l}46 \\
47 \\
48\end{array}$ & $\begin{array}{l}\text { II } \\
\text { II } \\
\text { II }\end{array}$ \\
\hline 19 & Chandanpur & $\begin{array}{l}\text { N } 19^{0} 53.459^{1} \\
\text { E } 85^{0} 49.76^{1}\end{array}$ & Bhargavi River & $\mathrm{CS}$ & 19 & 63 & I \\
\hline 22 & Mangrajpur & $\begin{array}{l}\mathrm{N} 19^{0} 50.126^{1} \\
\mathrm{E} 85^{0} 49.133^{1}\end{array}$ & Samang Lake & Sediment & 22 & 66 & II \\
\hline 25 & Baleswar & $\begin{array}{l}\mathrm{N} 19^{0} 49.651^{1} \\
\mathrm{E} 85^{0} 56.642^{1}\end{array}$ & Beach & Foreshore & 25 & 78 & III \\
\hline 28 & Banamalipur & $\begin{array}{l}\mathrm{N} 20^{0} 10.487^{1} \\
\text { E } 86^{0} 00.078^{1}\end{array}$ & $\begin{array}{l}\text { Kushabhadra } \\
\text { River }\end{array}$ & $\begin{array}{l}\text { CS } \\
\text { PB } \\
\text { NL } \\
\text { FP }\end{array}$ & $\begin{array}{l}28 \\
28 \\
28 \\
28\end{array}$ & $\begin{array}{l}88 \\
89 \\
90 \\
91\end{array}$ & $\begin{array}{l}\text { II } \\
\text { II } \\
\text { II } \\
\text { II }\end{array}$ \\
\hline 30 & Gop & $\begin{array}{l}\mathrm{N} 19^{0} 59.044^{1} \\
{\mathrm{E} 86^{0}}^{0} 00.784^{1}\end{array}$ & $\begin{array}{l}\text { Kushabhadra } \\
\text { River }\end{array}$ & $\begin{array}{l}\text { CS } \\
\text { PB } \\
\text { NL } \\
\text { FP }\end{array}$ & $\begin{array}{l}30 \\
30 \\
30 \\
30\end{array}$ & $\begin{array}{l}96 \\
97 \\
98 \\
99\end{array}$ & $\begin{array}{l}\text { I } \\
\text { I } \\
\text { I } \\
\text { I }\end{array}$ \\
\hline 32 & $\begin{array}{l}\text { Ramachandi } \\
\text { (Mouth 1) }\end{array}$ & $\begin{array}{l}\mathrm{N} 19^{0} 51.261^{1} \\
\mathrm{E} 86^{0} 03.620^{1}\end{array}$ & $\begin{array}{l}\text { Kushabhadra } \\
\text { River }\end{array}$ & $\begin{array}{l}\text { Estuary } \\
\text { Sediment-1 }\end{array}$ & 32 & 106 & II \\
\hline 36 & Chandrabagh & $\begin{array}{l}\text { N } 19^{0} 51.936^{1} \\
\text { E } 86^{0} 06.814^{1}\end{array}$ & Konark Beach & Foreshore & 36 & 122 & II \\
\hline
\end{tabular}

is common in these channels suggesting fresh water ecosystem.The Birupa river system (Table-3) comprises the northern part of the Mahanadi delta and also shows the dominance of algal growth and very little record of terrestrial pollen/spores except the rare occurrence of Pinus which was perhaps transported through air. Although mangroves are well established here in the estuarine distal part of LDP yet the rare occurrence of

\section{DISCUSSION}

The overall results show dominance of autochthonous algal (Spirogyra) organic matter in the UDP indicating low energy current in shallow lotic water during dry months and intermittent nutrient input from land during monsoon periods favoring primary and secondary productivity in the UDP. The organic matter deposited in 
UDP generally reflect autotrophs particularly algal productivity responding to nutrient input (nitrogen and phosphorous) from nearby agricultural fields indicated by Cerealia pollen and other terrestrial pollen grains. The middle deltaic part also shows AOM derived from algal mass and elements of back-mangrove zone. However, the lower deltaic area is represented by rounded amorphous organic matter of algal mass perhaps transported from upper deltaic area. An enlarged view of amorphous matter derived from algae (Spirogyra) reveal the basic spiral arrangement of pyrenoids in the present study. Earlier Transmission electron microscopy study revealed that many kerogens described as amorphous using standard microscopy actually consist of bundles of thin lamellae $(\sim 10-30 \mathrm{~nm})$ called ultralaminae, which represent insoluble biomacromolecules inherited from the outer cell walls of microalgae (Largeau et al., 1980; Derenne et al., 1993). The fine suspended sediment of the monsoonal river flow / floods deposited in the flood plain and marginal tidally influenced environments like grassy swamps, mangrove swamps, tidal flats and also in the deeper offshore environment which act as sinks for fine sediments. In the present study this environment was recorded in the MDP and LDP where the POMs are deposited under anoxic depositional environment. Residual estuarine circulation in non-monsoonal months also distributes fine sediments in the estuarine/deltaic environments of Mahanadi river distributaries.

The lighter fraction comprising BUD dominate in the Kuakhai-Kushbhadra tributary and Birupa-Chitrotpala tributary suggesting the seasonal runoff from nearby flood plains through these channels. The fluvial energy in the MDP and LDP is thus controlled by seasonal hydroperiods. The abundance of chlorophytic algae indicate shallow and slow-moving water indicating restricted runoff from land. When sediment supply to the shoreface is reduced, erosion will dominate on the shore and that will be accentuated when severe storms / cyclones hit the shore. During the last fifty years construction of a major dam (at Hirakud), a weir and barrage across the Mahanadi River have arrested sediment supply to the shoreface. Besides irregular / erratic rainfall pattern due to changes in monsoon dynamics, increased anthropogenic activities involving rampant deforestation in the hinterland and also other human-induced factors might have arrested sediment supply and transport to the shoreface. Under a regime of reduced fluvial sediment supply, the littoral drift system getting a potential sediment component from fluvial sand supply may be impoverished, which may hinder nearshore aggradational processes (Mohanti, 1990). A retrograding shoreline may result under these circumstances. Looking at the depositional scenario, could it be envisioned that the Late Holocene deltaic progradation under adundant sediment supply and sea level adjustment with a net gradual seaward shifting of the shoreline, is on the critical threshold of retrogression due to reduced sediment input under natural and anthropogenic forcings. If this is admitted, a staggering coastal erosion and landloss is implied (Mohanti, 1990).

The Mahanadi River mouth and its distributary Debi River mouth is prominent in the coastal margin. The geomorphic features associated with the present active tributaries of Mahanadi river like Debi-Kuakhai- Birupa that transport sediments from land to the near- shore deltaic system have been described by Mahalik (2006). Due to neotectonic activities the northern part of the Mahanadi delta system is now active (Kumar and Bhattacharya, 2003). The present study conforms with the above findings where high POM is preserved in the MDP and LDP in south and middle portion due to low fluvial energy allowing gradual settling of POM as compared to low POM deposition in the high fluvial current in the UDP and MDP in northern part except the estuarine area in the LDP. The weak sea water intrusion through the mouths of the distributary channels provide conducive saline anoxic bottom in the stratified near shore ecosystem for the preservation of POMs in the entire deltaic ecosystem.

The key control on the formation of marine petroleum source rocks (MPSRs) largely depends on the bottom water oxygenation which promotes POM preservation. Aerobic environments are characterized by organically lean sediments (0-3.0\% TOC) with Type III kerogen assemblages composed of relatively refractory land plant debris or highly degraded, marine-derived, amorphous organic matter (AOM). Their organic richness is largely dependent on sediment accumulation rate and proximity to sources of terrestrial organic matter supply. They produce mainly gas at maturation. Dysaerobic to anoxic environments are characterized by MPSR facies with high TOC values and Type II kerogen assemblages dominated by relatively lipid-rich AOM. These represent the classic 'black shale' source rocks and are the main source of petroleum in marine basins (Tyson, 1987). The present study provides an understanding of dysoxic to anoxic organic matter depositional environment in the deltaic distributary channels of the Mahanadi river which may serve as useful modern analogue in petroleum exploration while dealing with the older stratigraphic sequences.

\section{Conclusion}

1. The organic matter sediment supply and transport to the deltaic depocentre of Mahanadi River is concentrated in the Middle and lower deltaic part under the present day situation of delta building and space in the tropical setting of the shallow ecosystem in the east coast, India. 2. The POM is mainly derived from terrestrial land plants 
or fresh water algal mass Spirogyra.

3. The estuarine area harbouring extensive mangroves particularly in the northern part do not show rich assemblage of mangrove pollen indicating its foreshore transportation.

4. The study provides a modern analogue of organic matter depositional microenvironments in the deltaic channels of Mahanadi River system which enables to understand the nature of organic matter deposition and its preservation under the present state of geomorphology, vegetation, climate and sea level dynamics which is of great value for the meaningful interpretation of the origins and palaeo depositional environment of marine petroleum source rocks (MPSRs).

\section{ACKNOWLEDGEMENT}

Authors are thankful to Directors of their respective Institutes for encouragement and providing facilities during this collaborative work in the ONGC sponsored Project executed from Delta Studies Institute,

\section{Visakhpatnam.}

\section{REFERENCES}

Demaison, G. J and Moore, G.T. (1980). Anoxic environment and oil source bed genesis. Organic Geochemistry, 2 :9-31.

Derenne, S., Metzger, P. and Largeau, C. (1993). Similar morphological and chemical variations of Gloeocapsomorpha prisca in Ordovician sediments and cultured Botryococcus braunii as a response to changes in salinity. Organic Geochemistry, 19 (3): 299-313.

Emerson, S., Fisher, K., Reimers., C. and Heggie, D. (1985). Organic carbon dynamics and preservation in deep-sea sediments. Deep-Sea Res., 32: 1-21.

Erdtman, G. (1983). An Introduction to pollen analysis, Chronica Botanica Co., Waltham Mass, US.

Jagannathan, C.R., Ratnam, C., Baishya, N.C. and Dasgupta,

U. (1983). Geology of the offshore Mahanadi Basin : Petroleum Asia Journal. IV (4): 101- 104.

Kumar, K.V and Bhattacharya, A. (2003). Geological evolution of Mahanadi delta, Orissa using high resolution satellite data. Current Science, 85 (10) :1410-1412.

Largeau, C., Casadevall, E., Dif, D and Berkaloff, C. (1980). Renewable hydrocarbon production from the agla Botryococcus braunii. In: W. Paiz., J. Coombs and D. O. Hall, (Eds.) Energy from Biomass (pp. 653-658), Elsevier Appl. Sci. Publ., London.
Mahalik, N.K. (2006). A study of the morphological features and borehole cuttings in understanding the evolution and geological processes in Mahanadi delta, east coast of India. Jour. Geological Soc. of India. 67: 595-603.

Meijerink, A. M..J. (1982-83). Dynamic geomorphology ofMahanadi delta. ITC Journal Special Verstappen : 243250 .

Meyers, P.A. (1997). Organic geochemical proxies of paleoceanographic, paleolimnologic, and paleoclimatic processes. Organic Geochemistry, 27:213-250.

Mohanti, M. (1993). Coastal processes and management of the Mahanadi River deltaic complex, East Coast of India. Proceedings Coastal Zone 93, American Society of Civil Engineers, New York, U.S.A., 75 - 90.

Mohanti, M. (1990). Sea level rise : Background, Global concern and implications for Orissa Coast, India. In : G. V. Rajamanickam (Ed.), Sea level variation and its impact on coastal environment (pp.197 232) Tamil University Publication No. 131, Thanjavur, India,.

Nioyogi, D. (1975) Geology of the coastal plain in West Bengal and Orissa. Indian Journal of Earth Sciences, 2: 51-61.

Ray, S.B. (1988). Sedimentological and geochemical studies on the Mahanadi River estuary, East Coast of India. Unpublished Ph.D. thesis, Utkal University, Bhubaneswar, India, $204 \mathrm{pp}$

Ray, S.B. and Mohanti, M. (1989). Sedimentary processes in the Mahanadi River estuary, East Coast of India. Workshop on Coastal processes and coastal Quaternaries of Eastern India. Geological Survey of India, Eastern Region, Calcutta, pp. 28-29.

Sambasiva Rao, M, Nageshwara Rao, K and Vaidyanathan, R . (1978). Morphology and evolution of Mahanadi and Brahmini-Baitarni deltas Proc. Symp. Morphology and evolution of landforms. Dept. of Geology, University of Delhi, Delhi, 241-248.

Taylor, G..H., Teichmuller, M., Davis, A., Diessel, C. F. K., Littke, R and Robert, P. (1998). Organic Petrology. Gebruder Borntraeger, Berlin.

Tyson, R. V. (1987). The genesis and palynofacies characteristics of marine petroleum source rocks. In: Brooks, J. \& Fleet, A. J. (Eds.), Marine Petroleum Source Rocks, Geological Society, London, Special Pubication, 26, 47-67.

Tyson, R.V (1994). Sedimentary Org. matter. Organic facies and palynofacies. Chapman and Hall, London, 1-640.

Tyson, R.V. (1993). Palynofacies analysis In: Jenkins D (Ed.) Applied Micropalaeontology - Kluwer Academic , Dordrect 153-191.

Wright, L.D. (1985) River Deltas, In : R.A. Davis Jr. (Ed.), Coastal Sedimentary Environments Springer Verlag, New York, pp. 1-76. 